\title{
Interference-Aware Self-Optimizing Carrier Sensor for High Efficiency Wireless LANs in Dense Networks
}

\author{
II-Gu Lee ${ }^{*}$ \\ * Graduate School of Information Security, KAIST \\ 291 Daehak-ro, Yuseong-gu, Daejeon, \\ †PHY Research Group, NEWRATEK \\ 433, 193 Moonji-ro, Yuseong-gu, Daejeon, \\ Republic of Korea 305-701 \\ Iglee9@kaist.ac.kr
}

\author{
Myungchul Kim \\ Graduate School of Information Security, KAIST \\ 291 Daehak-ro, Yuseong-gu, Daejeon, \\ Republic of Korea 305-701 \\ mck@kaist.ac.kr
}

\begin{abstract}
Wireless local area networks (WLANs) can adopt dynamic channel access technologies such as dynamic bandwidth or channel hopping schemes in order to avoid interference for better link quality. However, in dense networks, the dynamic channel access leads to a higher probability of adjacent channel interference (ACI). The efficiency of IEEE 802.11-based WLANs using multi-channel and wide dynamic ranges is thus severely degraded by ACIs in dense networks. In this paper, we analyze the ACI effect on WLANs and propose an interference-aware self-optimizing carrier sensor design that incorporates a multichannel multi-level carrier sense and adaptive initial gain control scheme. This scheme controls carrier sensing thresholds in each band for multi-level sensors, as well as initial gains for amplifiers. The proposed scheme reduces false carrier sensing and avoids saturation of amplifiers while simultaneously improving the dynamic range of the receiver. Our prototype evaluation results demonstrate that the proposed scheme can improve the dynamic range of the receiver by approximately $45 \mathrm{~dB}$ and $30 \mathrm{~dB}$ for a low data rate and a high data rate mode, respectively, compared with the conventional receiver designs. Furthermore, network emulation results demonstrate that the proposed scheme can improve the average throughput and latency by approximately $32 \%(24 \%)$ and $41 \%(43 \%)$, respectively, compared with the conventional receiver designs (and channel hopping techniques) in dynamically varying interfered channel conditions.
\end{abstract}

\section{Categories and Subject Descriptors}

C.2.1 [Computer-Communication Networks]: Network Architecture and Design - Wireless communication.

\section{General Terms}

Algorithm, Design, Experimentation, Performance.

Permission to make digital or hard copies of all or part of this work for personal or classroom use is granted without fee provided that copies are not made or distributed for profit or commercial advantage and that copies bear this notice and the full citation on the first page. To copy otherwise, to republish, to post on servers or to redistribute to lists, requires prior specific permission and/or a fee.

MOBIQUITOUS 2015, July 22-24, Coimbra, Portugal

Copyright (c) 2015 ICST 978-1-63190-072-3

DOI 10.4108/eai.11-8-2015.151112

\section{Keywords}

Adjacent channel interference, carrier sense, gain control, channel hopping, WLAN, jamming, security.

\section{INTRODUCTION}

Wireless local area network (WLAN) technologies are an essential feature of everyday life: they are used for home networking, smart mobile devices, network infrastructures, and much more. As more and more wireless devices are connected and wireless access points (APs) are densely deployed in the scarce frequency spectrum, the failure probability of packet transmission is expected to increase due to interference from other devices. Because the $2.4 \mathrm{GHz}$ band is already congested and the 5 $\mathrm{GHz}$ band will be congested soon [1], the wireless environment might suffer severe interference from unintentional wireless devices or intentional jammers [2, 3]. Furthermore, the increasing deployment of APs for wireless fidelity (WiFi) service leads to interference from neighboring APs and mobile nodes, thus noticeably degrading the network performance and damaging the network security and quality of service (QoS) [4].

Traditionally, WLAN technologies have increased the data rate by extending the channel bandwidth from $20 / 40 \mathrm{MHz}$ in the IEEE $802.11 \mathrm{a} / \mathrm{b} / \mathrm{g} / \mathrm{n}$ standards to $80 / 160 \mathrm{MHz}$ in the IEEE $802.11 \mathrm{ac}$ standards [5]. However, the wider channel bandwidth is challenging because there may be legacy devices in the same or neighboring basic service sets (BSSs) and there may be heterogeneous mobile devices in adjacent channels. Because many APs are installed without considering the existing nearby APs, their spectrums can overlap and cause interference between neighbor BSSs, potentially becoming a serious problem. It is expected that the number of overlapped BSSs (OBSSs) in IEEE $802.11 \mathrm{n} / \mathrm{ac}$ will become greater than those in IEEE $802.11 \mathrm{a} / \mathrm{b} / \mathrm{g}$ due to both having wider bandwidths and increases in the number of WLAN devices. This situation may lead to consistent collisions or degradation of the signal quality. It is thus very challenging to guarantee the QoS requirements in wireless environments because wireless links might have dynamically varying interference.

Several studies have investigated the adjacent channel interference (ACI) effect, and several approaches to use new protection mechanisms in WLAN systems have been reported. However, their scope was confined to demonstrating deteriorated performance due to ACI for the legacy $11 \mathrm{a} / \mathrm{b} / \mathrm{g} / \mathrm{n}$ wireless LAN systems [6,7], while other studies have suggested using a different channel via channel hopping or AP based channel assignment techniques $[8,9,10]$. Some of the new protection 
mechanisms require modification of the current standard $[9,10$, 11]. In this paper, we analyze the ACI effect on the carrier sense (CS) and automatic gain control (AGC) of WLANs, and we describe the adverse effects of ACI on the efficiency of WLANs using wider channel bandwidths and overlapped service ranges. In order to address these problems, we present an interference-aware self-optimizing (IASO) carrier sensor jointly optimizing initial gain and multi-channel multi-level carrier sensing thresholds. This technique reduces false carrier sensing and avoids saturation of amplifiers while simultaneously improving the dynamic range of the receiver. Because the WLAN spectrum has been diversified and overlapped, the traditional receiver design and channel hopping technique are no longer effective in preventing losses in system efficiency.

The proposed ACI mitigation scheme has two key features: it employs a multi-channel and multi-level carrier sensing design in order to reduce false carrier sensing and it has an adaptive initial gain control with amplifiers and filters in order to improve the dynamic range. The proposed IASO scheme provides substantial improvements in terms of the dynamic range, throughput, and latency of the receiver utilizing a cross layer design. The experimental evaluation results confirm the significantly improved performance of the IASO scheme compared with the conventional schemes under dynamically varying interfered channel conditions.

This paper makes the following three key contributions:

- This is the first work to mitigate the ACI effect in WLANs through an interference-aware self-optimizing carrier sensor design jointly using initial gain control and multi-channel multi-level carrier sense.

- The proposed scheme is implemented and evaluated in a field programmable gate array (FPGA) prototype, and in a cycle true and bit true emulation platform, consistent with the commercial hardware, in order to demonstrate its performance in realistic environments.

- The IASO scheme achieves substantial improvements in the dynamic range, throughput, and latency of receiver with simple modifications while maintaining compatibility with the existing WLAN standards.

The remainder of this paper is organized as follows. In Section 2, we describe the ACI effect and review related works. In Section 3, we analyze the ACI problem and its causes. In Section 4, we propose an IASO scheme. In Section 5, the evaluation results are presented, and finally Section 6 draws conclusions.

\section{ACI effect and related work}

In this section, the ACI effect and related works are described.

\subsection{Carrier sense and gain control}

Carrier sensing has an important function in the carrier sense multiple access with collision avoidance (CSMA/CA) protocol for WLANs. On the transmitter side, carrier sensing is used as an indicator for collision avoidance by sensing the medium for a fixed duration when it needs to transmit packets. In the IEEE 802.11 standard, the carrier sensing scheme is based on clear channel assessment (CCA) and the network allocation vector (NAV). The CCA manages the carrier sensing including energy detection and signal detection at the physical layer, and the NAV is the header information-based sensing mechanism at the medium access control (MAC) layer. The medium is determined to be idle only when both the CCA and NAV indicate that the channel is idle. There have been several studies on mitigating the interference effect by adapting the CCA level based on statistical error information [12]. The CCA adaptation techniques are approaches to obtain more transmission opportunities by reducing the sensitivity of CCA, but they might cause higher probability of interference with other devices [13].

On the receiver side, carrier sensing is used to detect the start of an incoming signal. As illustrated in Figure 1, the conventional receiver front-end consists of an analog amplifier, carrier sensor, AGC circuit, and loop filter circuit $[14,15]$. The carrier sensor and gain controller use the incoming signal from the ADC output to detect the signal and adjust the signal level to the dynamic range. In order to process the incoming signal in a timely manner, the carrier sensor in the receiver is implemented in the physical layer. The baseband processor detects an incoming signal from the analog-to-digital converter (ADC) based on a preamble or on energy detection. If a carrier sensor detects an incoming signal, the receiver controls the gain. The gain control function should be implemented in wireless devices where the incoming received signal can vary over a wide dynamic range. In order to detect and decode the received signal with an improved signal-to-noise ratio (SNR), the AGC circuit can be used to adjust and track the average power close to the desired level. The AGC circuit tracks the power variation caused by the channel variation and adjusts the received signal power to an optimal SNR level within the dynamic range of various signal processing components in the received signal path. However, if there are interferers in a neighbor BSS or adjacent channel, the conventional schemes might suffer performance degradation due to false carrier sensing and a limited dynamic range of the receiver because they have not considered the ACI effect.

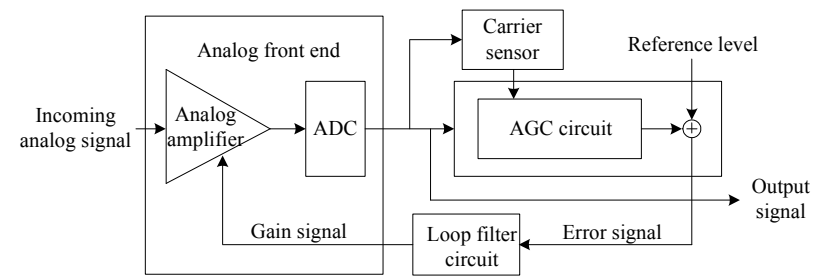

Figure 1. Conventional carrier sensor and gain controller.

\subsection{Channel hopping}

The IEEE $802.11 \mathrm{~h}$ standard defines the dynamic frequency selection (DFS) mechanism in order to avoid interference from radars and other WLAN devices [16]. The DFS mechanism allows an AP and its associated stations to dynamically switch to another channel in order to avoid interference. However, the frequency hopping schemes have several limitations. First, they are not effective for dynamically varying interference: they might show performance degradation caused by the fixed decision threshold to switch the channel even though the interference interval and level may vary in all channels. Second, the frequency hopping schemes increase the overhead for control frame transactions, channel switching settling times, and waiting time for sufficient error statistics. Third, they do not guarantee clean co-channels or adjacent channels in the next hop.

\section{ACI problem analysis}

In this section, the ACI problem and its causes are analyzed from network operation, system design, and standard perspectives. 


\subsection{Network operation and system design}

From a network operation perspective, ACI is caused by the packet exchange in partially overlapping channels due to the uncoordinated AP distribution and inappropriate primary channel allocation, which is accessed using carrier sensing for coexistence and backward compatibility with older WLAN standards. It is more difficult to select a primary channel that is common to all overlapping networks and to avoid the effect of interference because wider channel usage means a greater probability of cochannel or adjacent channel operation in the scarce spectrum frequency. There have been several proposals to improve the scheduling capabilities of APs and thereby improve average throughput and maintain fairness, because the enhanced distributed channel access (EDCA) mechanism in multiple BSS networks leads to poor average throughput and high airtime occupation [17]. Furthermore, the self-organizing network (SON) or AP scheduling mechanisms require standardized features such as information exchange between neighboring APs [9, 18]. Therefore, the SON and AP scheduling schemes are beyond the scope of this paper because they are not possible in the current WLAN standards.

From a design viewpoint, $\mathrm{ACI}$ is caused by the nonlinearity of the analog power amplifiers and the imperfectness of the filters. Even though two mobile nodes use two different frequency channels, interference in the form of leakage power can bleed into the other channel. Strong ACIs that fall into the in-band of a neighbor receiver can severely degrade the dynamic range, throughput, and latency.

\subsection{IEEE 802.11 standard}

In IEEE 802.11a devices, the legacy RTS/CTS and virtual carrier sense are only respected on the primary $20 \mathrm{MHz}$ channel: the RTS/CTS rules do not consider the hidden node problem in secondary channels. Therefore, it is expected that the hidden node problem becomes more severe in IEEE 802.11n/ac standards using wider channels because wider bandwidths require improved interference mitigation in the non-primary channels. IEEE 802.11 n devices can manage overlapping network problems by choosing different channels or primary channels that match the neighbor if overlapping conditions are unavoidable. However, this becomes significantly more difficult in IEEE 802.11 ac devices because the wider channel transmission means there is a greater probability of overlapping channel operation as well as increasing difficulty in choosing a primary channel common to all overlapping networks. Because data are transmitted using a wider bandwidth and dynamic channel access, interference and coexistence problems may occur between communicating nodes that support various standards. In order to support high throughput services with high reliability, IEEE 802.11ac adopts wider channel transmission up to $160 \mathrm{MHz}$ bandwidth and mitigates cochannel interference problems with a dynamic bandwidth mechanism.

IEEE 802.11ac devices support a static channel access and a dynamic channel access. The static channel access mechanism is an extended version of the IEEE 802.11n channel access for 40 $\mathrm{MHz}$ bandwidth. A station performs a back-off procedure based on CCA in the primary $20 \mathrm{MHz}$ channel. In the static channel allocation presented in Figure 2(a), if all non-primary channels are sensed free for a point coordination function inter-frame space (PIFS) period when the back-off counter reaches zero after an arbitration inter-frame space (AIFS), the transmitter can send in the $80 \mathrm{MHz}$ bandwidth. However, if the station determines that one of the secondary channels is busy, it can restart the back-off procedure. In the dynamic channel access mechanism in Figure 2(b), if one of the secondary channels is busy, a narrower channel transmission is permitted including the primary channel.
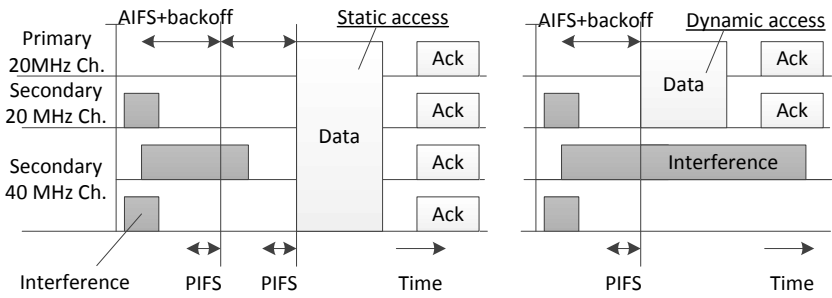

Figure 2. CSMA-based medium access when ACI affects the transmitter: (a) static channel access and (b) dynamic channel access.
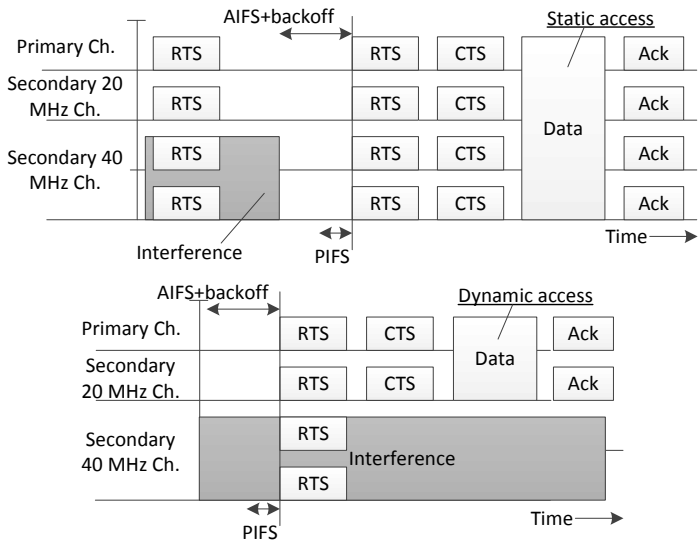

Figure 3. CSMA-based medium access when ACI affects the receiver: (a) static channel access and (b) dynamic channel access.

As illustrated in Figure 3(a), if a static bandwidth mechanism is used, data frames are affected by interference in the secondary 40 $\mathrm{MHz}$ channel. In contrast, in Figure 3(b), if the transmitter does not sense the secondary $40 \mathrm{MHz}$ channel and the receiver is affected by the interference, they can change the transmission bandwidth using an RTS/CTS handshake. However, even though the WLAN standards have attempted to improve the co-channel interference problem using the dynamic bandwidth mechanism, the wider channel bandwidth and dynamic channel access result in a higher probability of adjacent channel interference. This means a primary channel transmission might begin while a secondary channel transmission is in progress. This process results in wireless devices being affected by the ACI more frequently. Therefore, there is a need for techniques that optimize the receiver by mitigating the effect of interference from neighbor nodes in order to maintain high throughput with high reliability.

Furthermore, WLAN technologies have been evolving to use wider channel bandwidths for IEEE $802.11 \mathrm{ac}$ [5] in the $2.4 / 5 \mathrm{GHz}$ frequencies and they support a wide range for IEEE 802.11af/ah in the sub-1 GHz frequency $[19,20]$. Because there is a high probability that two or more different types of BSSs will overlap in adjacent channels of the wider bands and in the extended range where the range can be up to $1 \mathrm{~km}$, this issue has emerged as an important challenge. Furthermore, because the emergence of the 
IEEE 802.11ax high-efficiency WLAN (HEW) [21, 22] aims to achieve a substantial enhancement in throughput and latency in the real world of highly dense network environments with large numbers of APs and stations, the issue will become increasingly important.

\section{Interference-aware self-optimization}

This section describes the receiver design for the proposed IASO scheme. We then present an exemplary network operation using the proposed scheme.

\subsection{IASO carrier sensor design}

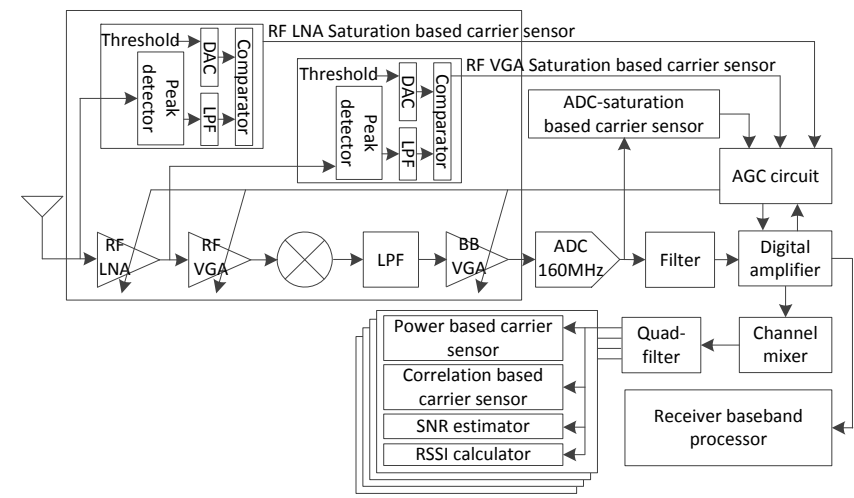

Figure 4. IASO carrier sensor design.

Figure 4 illustrates the receiver front-end blocks. The receiver has two-stage analog saturation-based carrier sensors for wideband signals and a digital saturation-based carrier sensor for narrowband signals. The wideband carrier sensor can detect a large ACI state in RF/analog circuits, while the digital baseband cannot detect it because the digital input is narrowband after the analog/digital lowpass filtering. Thus, these analog/digital saturation-based carrier sensors allow the digital baseband processor to determine whether the strength of a received signal is dominated by an out-of-band interference signal or by an in-band desired signal. The two analog saturation-based carrier sensors are integrated into a RF low noise amplifier (LNA) and a RF variable gain amplifier (VGA). Each analog saturation-based carrier sensor consists of a peak detector placed near the RF circuit, a low pass filter (LPF), and a comparator. A digital-to-analog converter (DAC) is used to set the threshold in the comparator according to the programmable register. The threshold for each of the two RF saturation based carrier sensors is programmed through a serialto-parallel interface (SPI) using a digital baseband processor. Each saturation based carrier sensor provides one digital flag output to the digital baseband processor. If the RF LNA or RF VGA saturation flag is set, it indicates that the output after the RF LNA or RF VGA is greater than the threshold programmed through the SPI, respectively.

In the digital baseband, for a strong in-band signal and fast gain control, the ADC-saturation based carrier sensor monitors the ADC output in order to verify if the incoming signal is saturated or not. For reliable detection of the ADC saturation, consecutive incoming samples are used. If the accumulative saturated sample counts though the I and Q paths of the ADC output are larger than a specified threshold, then this circuit informs the AGC circuit that the ADC is saturated. The other types of carrier sensing, such as using signal power and correlation, are completed in the power and correlation based carrier sensors, respectively. The correlation-based carrier sensors detect the correlation peak utilizing the known preamble sequence or repetition property. The signal power, correlation, SNR, and received signal strength indicator (RSSI) are measured for each signal band. The received signal from an antenna is fed into the AGC circuit in order to adjust the incoming signal strength to the target reference power. The incoming signal power is measured from the output of the $\mathrm{ADC}$ and the optimal gain is calculated and updated in the AGC circuit. The gain control is implemented in three stages from the $\mathrm{RF}$ input to the digital front end: a $32 \mathrm{~dB}$ RF LNA with three modes in $16 \mathrm{~dB}$ steps, a $64 \mathrm{~dB}$ analog baseband VGA in $2 \mathrm{~dB}$ steps, and a $12 \mathrm{~dB}$ digital baseband amplifier in $3 \mathrm{~dB}$ steps. All amplifiers are controlled using the AGC circuit of the digital baseband.

\subsection{IASO operation}

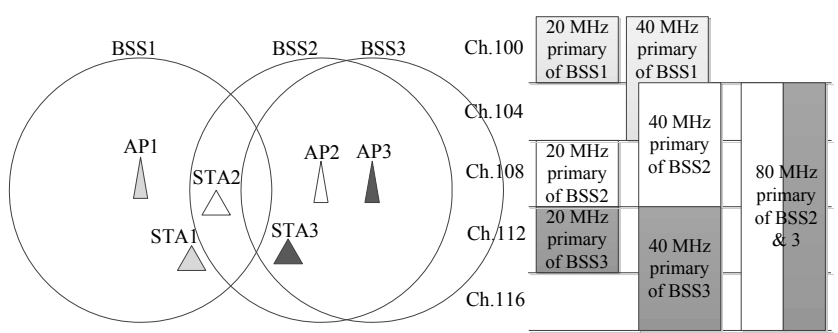

Figure 5. An ACI scenario: (a) network configuration and (b) primary channel allocation of APs.

Table 1. Channel parameters for BSSs

\begin{tabular}{|c|c|c|c|}
\hline Category & BSS1 & BSS2 & BSS3 \\
\hline Standard & $802.11 \mathrm{n}$ & $802.11 \mathrm{ac}$ & $802.11 \mathrm{ac}$ \\
\hline $\begin{array}{c}\text { Supported } \\
\text { bandwidth } \\
{[\mathrm{MHz}]}\end{array}$ & $20 / 40$ & $20 / 40 / 80$ & $20 / 40 / 80$ \\
\hline Channel & 100,104 & $\begin{array}{c}104,108, \\
112,116\end{array}$ & $\begin{array}{c}104,108, \\
112,116\end{array}$ \\
\hline Primary channel & 100 & 108 & 112 \\
\hline AP index & 1 & 2 & 3 \\
\hline $\begin{array}{c}\text { Associated STA } \\
\text { index }\end{array}$ & 1 & 2 & 3 \\
\hline
\end{tabular}

Figure 5(a) presents an example of an ACI scenario. There are three different BSSs that are allocated to different primary channels, as described in Table 1 . BSS2 supports 20/40/80 MHz bandwidth because AP2 has IEEE 802.11ac capability. BSS1 and BSS3 use adjacent channels as in-bands and bands adjacent to BSS2, respectively. In BSS1 and BSS2, AP1 can cause frequent interferences at STA2 when it transmits packets. Even though AP3 and STA3 use different channels to that used by AP2, their transmission power can bleed over the primary channel of AP2. However, STA2 may not decode weak signals using the $40 \mathrm{MHz}$ or $80 \mathrm{MHz}$ bandwidth due to the interference from AP1, STA1, and STA3, or it may fail to receive or transmit frames due to false carrier sensing and signal saturation. Figure 5(b) illustrates the primary channel allocation in the scenario for three APs. Multiple BSSs can use dynamic bandwidth to share access to the same wide channels. BSS1 and BSS2 have different primary channels, but they share a partially overlapped $40 \mathrm{MHz}$ band. BSS2 and 


\begin{tabular}{|c|c|c|c|c|c|c|c|c|c|c|c|c|c|}
\hline \multirow{3}{*}{$\begin{array}{l}\text { Adjacent channel (AC) } \\
\text { In-band channel (IC) }\end{array}$} & \multirow{2}{*}{\multicolumn{10}{|c|}{ Adjacent channel interference }} & & & \\
\hline & & & & & & & & & & & & & \\
\hline & & & & \multirow{2}{*}{\multicolumn{2}{|c|}{ L-STF }} & \multirow{2}{*}{ L-LTF } & \multirow{2}{*}{$\begin{array}{c}\text { L- } \\
\text { SIG }\end{array}$} & \multirow{2}{*}{$\begin{array}{l}\text { VHT- } \\
\text { SIGA }\end{array}$} & \multirow{2}{*}{\begin{tabular}{|l|} 
VHT- \\
STF \\
\end{tabular}} & \multirow{2}{*}{$\begin{array}{l}\text { VHT- } \\
\text { LTF }\end{array}$} & & & \\
\hline \multicolumn{4}{|l|}{ Receiver state } & & & & & & & & & & \\
\hline $\begin{array}{l}\text { Carrier sensing } \\
\text { for } I C \text { and } A C\end{array}$ & $\begin{array}{c}\text { Carrier } \\
\text { sensed } \\
\text { in } A C\end{array}$ & $\begin{array}{l}\text { Optimized } \\
\text { (Ts and } \\
\text { Gi) }\end{array}$ & $\begin{array}{c}\text { Carrier sensing for } \\
\text { IC \& carrier sensed } \\
\text { in AC }\end{array}$ & \multirow{2}{*}{\multicolumn{2}{|c|}{ 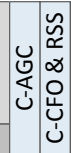 }} & \multirow{2}{*}{ 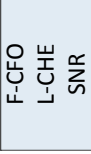 } & \multirow{2}{*}{\multicolumn{2}{|c|}{ 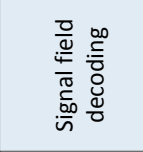 }} & \multirow[t]{2}{*}{ 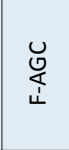 } & \multirow[t]{2}{*}{ 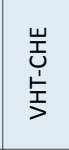 } & \multirow[t]{2}{*}{ 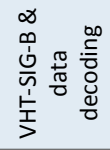 } & $\begin{array}{l}\text { Optimized } \\
\text { (Ts and Gi) }\end{array}$ & $\begin{array}{l}\text { Carrier sensing } \\
\text { for } I C \text { and } A C\end{array}$ \\
\hline \multicolumn{4}{|c|}{ Multi-channel multi-level carrier sensing } & & & & & & & & & Multi-channe & alti-level carrier sensing \\
\hline
\end{tabular}

Figure 7. Receiving processing procedures.

BSS3 do not share their primary $20 \mathrm{MHz}$ or $40 \mathrm{MHz}$ channels, which are independently transmitted, but they share the same 80 $\mathrm{MHz}$ channel for transmission.

Figure 6 presents the dynamic bandwidth operation of IEEE 802.11 ac devices in the multiple BSS network. Boxes numbered 1,2 , and 3 represent the $\mathrm{BSS} 1, \mathrm{BSS} 2$, and BSS3, respectively. The IEEE 802.11ac standard allows different networks to share a wide bandwidth channel much more efficiently than IEEE $802.11 \mathrm{n}$. However, this causes more frequent ACIs among multiple networks. For example, in T1 time, STA2 receives interference from AP3 or STA3, but the interference is not detected by AP2 because the legacy RTS/CTS protection mechanism is only effective for the hidden node problem of the OBSS over the primary channel. Therefore, bandwidth signaling is added to the duplicated RTS and CTS frames in the dynamic channel access mechanism. AP2 sends an RTS with the bandwidth of the intended transmission, and STA2 sends a CTS response with a bandwidth of clear channels. Based on the clear channel information, AP2 only sends a data frame on the clear channels. Therefore, as seen in Figure 3(b), AP2 transmits an RTS frame using $80 \mathrm{MHz}$ bandwidth indication and STA2 sends a CTS frame with $40 \mathrm{MHz}$ bandwidth indication because channels 112 and 116 from AP3 and STA3 are interfering. Then, AP2 only sends a data frame through channels 104 and 108.

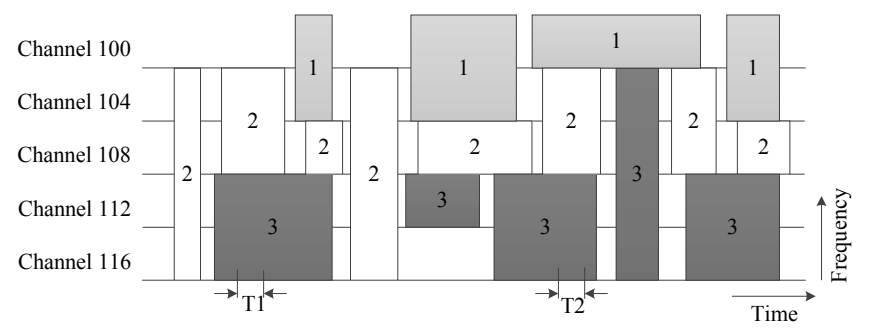

Figure 6. Dynamic bandwidth transmission.

Figure 7 illustrates the IASO process utilizing multi-channel multi-level carrier sensing and initial gain control. The initial gain settings should be as large as possible in order to maximize the service coverage by receiving a very weak signal. However, in the maximum initial gain settings, the incoming signals through the RF and analog components may be saturated by strong ACIs. Thus, the receiver should determine whether the ACIs cause saturation and whether they exist, and it should control the initial gain settings through per-frame detection of ACI. The receiver can receive a data frame after adjusting the initial gain and sensing thresholds based on the multi-channel multi-level carrier sensors.
The in-band channel signal presents the frame structure for IEEE $802.11 \mathrm{ac}$ [5]. The frame contains a header for the signal detection, gain control, synchronization, channel estimation, and signal information. The receiver decodes the received signal in the order of legacy short training field (L-STF), legacy long training field (L-LTF), legacy signal field (L-SIG), VHT-SIG-A, VHT-STF, VHT-LTF, VHT-SIG-B, and data fields. The L-STF is intended for carrier sensing, AGC, coarse carrier frequency offset (C-CFO) acquisition, and symbol synchronization; the L-LTF is designed for fine carrier frequency offset (F-CFO) acquisition and legacy channel estimation (L-CHE). The signal fields include the rate, length, and transmission mode information for the receiver to decode the received frame. The receiver can detect the type of frame based on the phase rotation of the VHT signal fields. The VHT-STF is used for fine AGC (F-AGC), and the VHT-LTF is used for the VHT channel estimation (VHT-CHE). The VHTSIG-B is used to signal multi-user (MU) specific information. In the data fields, the receiver decodes and tracks the incoming symbols.

The receiver can conduct analog and digital gain control using saturation information from multi-level wideband/narrowband saturation-based carrier sensors of RF LNA, RF VGA, and ADC. Each carrier sensor has its own detection threshold and detection band. Thus, the receiver can differentiate the control flow for interference and the desired signal detection. In the idle channel state, the receiver is sensing in both primary and adjacent channels. If one of the adjacent channels is sensed, the receiver optimizes the sensing thresholds $\left(\mathrm{T}_{\mathrm{s}}\right)$ and initial gain settings $\left(\mathrm{G}_{\mathrm{i}}\right)$ and continues to sense in the primary channel. The gain controller of the digital baseband can detect the saturation state caused by a large ACI and avoid the state by rapidly reducing the gain with large gain even after filtering out the interference. Furthermore, it can control the initial gain settings in order to avoid the signal saturation by a strong ACI for the next incoming frames. After sensing the desired signal, the receiver processes the frame, and then optimizes $\mathrm{T}_{\mathrm{s}}$ and $\mathrm{G}_{\mathrm{i}}$.

In addition, even though the gain control process is not completed in the L-STF for VHT frames due to the large ACI, it can be performed in the fine gain control stage of the VHT-STF. Because the legacy compatible part is BPSK-modulated and the VHT compatible part might be high order-modulated, the receiver has SNR headroom for the legacy compatible part in rate adaptive WiFi devices. Therefore, the receiver becomes more robust because the interference effect is mitigated again in the VHTSTF. In order to achieve more robustness, if the guard bands are allocated to the secondary channels, the receiver can achieve additional dynamic range improvement in the T2 time in Figure 6. Even though this guard band allocation has a limitation in that 

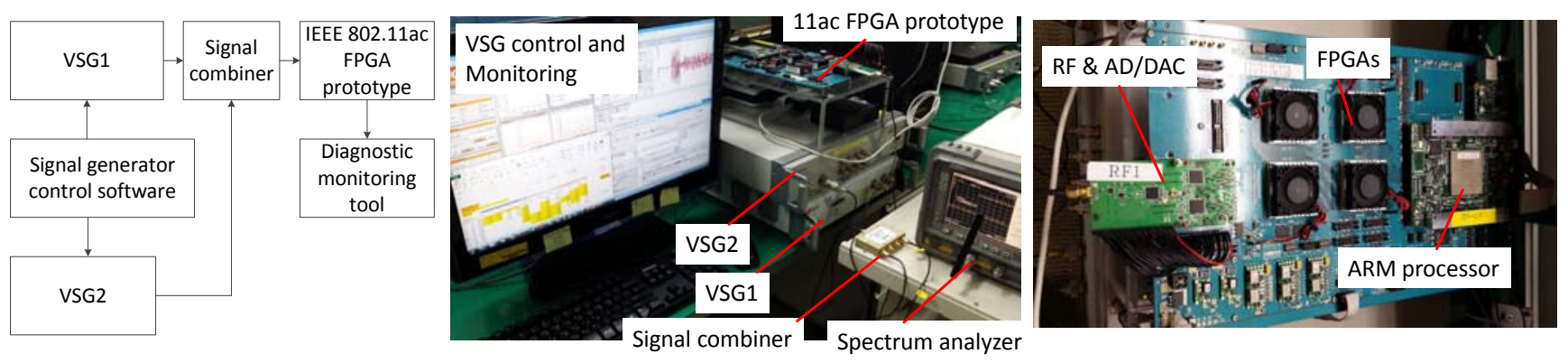

Figure 8. Test configuration (left), experimental setup (middle), and IEEE 802.11ac prototype system (right).

only a single side guard band can be allocated in the T2 time case, which has a dual ACI effect, it can mitigate the ACI from BSS1.

\section{Performance evaluation}

In this section, the proposed scheme is evaluated and compared with the conventional schemes. In order to verify the functionality and performance of the proposed IASO scheme, we implemented an IEEE 802.11ac FPGA prototype, and a cycle true and bit true emulation platform, which is the same as the commercial hardware. The hardware was verified in a FPGA with a commercial AP and station, and the circuits were implemented in an ASIC for commercial products. For performance comparisons, we defined three metrics of interest: dynamic range, per packet throughput, and per packet latency of receiver. We measured the dynamic range of the receiver under various ACI conditions in the prototype. We also proposed the inclusion of the PHY and MAC system emulation as tools to assess the gains of those metrics and proposed a set of test scenarios to capture the effect of the interference level on the carrier sense and gain control. The performance curve for the IASO scheme described in the previous sections is presented in order to demonstrate how the proposed scheme outperforms the existing schemes.

\subsection{FPGA prototype experiment}

As shown in Figure 8, the experimental setup consists of an IEEE 802.11ac FPGA prototype, two vector signal generators (VSGs), signal generator control software, and a diagnostic monitoring tool. The FPGA prototype satisfies the functionalities and performance requirements of the IEEE 802.11ac standard. A spectrum analyzer was used to verify the existence of other interference in the channel. There are two VSGs for the desired signal and interference. The output of the VSG1 for the desired signal was connected to the FPGA prototype, and an additional signal generator (VSG2) was added in order to provide an interfering signal for the adjacent channel test through the signal combiner. This interfering signal generator functions as a neighbor node that sends IEEE 802.11ac compliant frames in an adjacent channel. When setting the levels on the VSGs, the gain losses from cables and combiners are considered. The performance and functionalities can be observed through monitoring software or a chip scope. The VSG1 for the desired signal sends 10,000 packets at a specified power level. An internal cyclic redundancy check (CRC) counter in the digital baseband is used to calculate the packet error rate because the probability of undetected erroneous packets is very small in the 32-bit CRC. The packet error rate (PER) at a given input power level ensures that the receiver is robust to noise, interference, distortion, and other factors that might affect communication at the level. The PER typically degrades at both very low and very high signal powers due to the limitation of the SNR and linearity, respectively.

The right side of Figure 8 also illustrates the developed IEEE 802.11ac prototype system, which consists of a RF IC, DAC, ADC, four Xilinx Virtex6 FPGAs, and an ARM Cortex-A5 processor. The four FPGAs and ARM processor are programmed for the functionalities of the IEEE 802.11 ac PHY/MAC hardware and MAC software, respectively, which was verified using commercial products in order to meet the requirements of the WiFi standards. This prototype was developed to verify the functionality and performance of the digital baseband PHY/MAC system before taping it out for silicon. The circuits targeted in the prototype were designed to support IEEE $802.11 \mathrm{a} / \mathrm{b} / \mathrm{g} / \mathrm{n} / \mathrm{ac}$ with a single antenna in order to support a high data rate of up to 433 Mbps in the $2.4 \mathrm{GHz}$ and $5 \mathrm{GHz}$ ISM bands. The RF IC is connected to the digital baseband through the ADC and DAC ICs operating at a $160 \mathrm{MHz}$ sampling rate. The digital baseband controls the RF transceiver in order to change the system parameters such as the TX/RX mode, gain, channel frequency, and filter mode through external pins or SPI.

In this evaluation, the minimum sensitivity is defined as the lowest power level at which a receiver can achieve 10\% PER for a frame length of 4,096 bytes. Higher order modulation schemes such as 256 QAM require a higher SNR at the receiver in order to achieve the same frame error rates as more robust schemes, such as BPSK. Sensitivity describes the performance of a receiver in a region with very low intensity signals. The IEEE 802.11 standard specifies that $10 \%$ PER should be achieved for a maximum input level of $-30 \mathrm{dBm}$ in the $5 \mathrm{GHz}$ band. The maximum input level characterizes the performance of a receiver in scenarios where the received signal strength is high. This measurement ensures that the receiver functions as expected when it is located close to the transmitter. In these cases, although the received signal SNR might be quite high, the high input power of the received signal could potentially drive the receiver's front-end components into compression, which distorts the signal. The maximum input level is an important figure of merit when the receiver is very close to an AP. Using these definitions, the dynamic range of the receiver is strictly described as the difference between the minimum input sensitivity and the maximum input level. 
The adjacent channel rejection (ACR) represents the ability to receive a desired signal while a strong interfering signal is present at an adjacent channel. It is specified as the power difference between the interfering channel and the desired channel. The ACR measurement characterizes the receiver's performance in a scenario where a relatively high power signal is present in the channel directly adjacent to the reference channel. The IEEE 802.11 standard specifies that a receiver should meet a PER of less than $10 \%$ at the power of the interfering signal, which is equal to the channel rejection values for the different modulation and coding schemes (MCSs). For example, ACR for MCS0 and MCS 8 in $20 \mathrm{MHz}$ bandwidth are $16 \mathrm{~dB}$ and $-9 \mathrm{~dB}$, respectively. The ACR test is performed by setting the desired signal's strength $3 \mathrm{~dB}$ above the sensitivity level, and it is applied by the interfering signal with a specified power of the same bandwidth in the adjacent channel. For example, the first signal generator transmits the desired signal at a level $3 \mathrm{~dB}$ above the minimum sensitivity level, and the second signal generator transmits the interfering signal at a level $16 \mathrm{~dB}$ above the minimum sensitivity level for the BPSK and $1 / 2$ code rate case. The interferer is a conformant OFDM signal that is not synchronized with the desired signal with more than a $50 \%$ duty cycle. The PER is then measured while raising the power of the interfering signals until $10 \%$ PER is met in the presence of strong interfering signals in an adjacent channel.

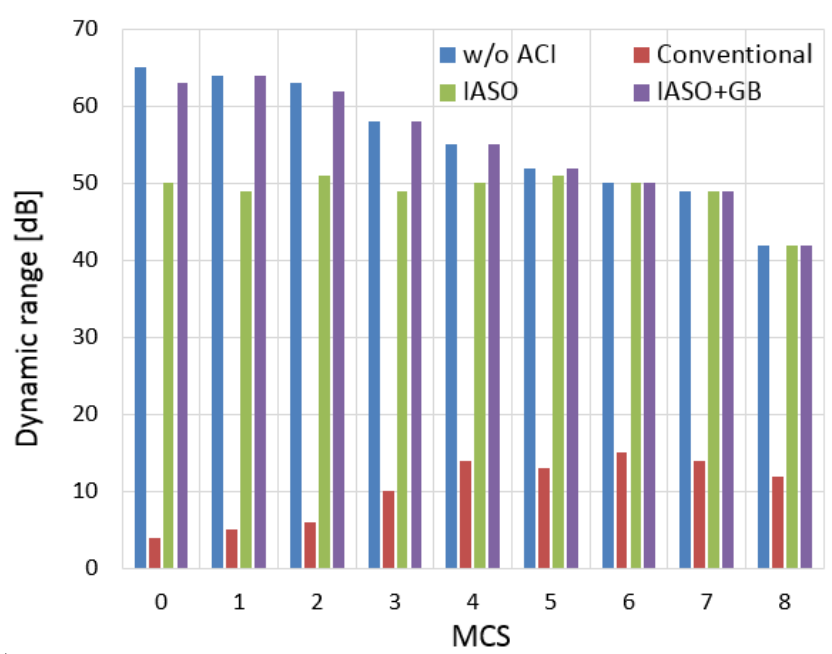

Figure 9. Dynamic range of the receiver.

When the baseband processor detects the presence of a strong ACI signal from the wideband carrier sensors of the RF/analog part and narrowband carrier sensor of the digital baseband, the AGC circuit changes the gain settings of the amplifiers at the receiver. The digital multi-channel multi-level carrier sensors further improve the dynamic range by adjusting the sensing thresholds. As a result, as seen in Figure 9, the proposed IASO scheme can improve the dynamic range of the receiver by approximately $30 \mathrm{~dB}$ and $45 \mathrm{~dB}$ for high MCS and low MCS, respectively, compared with the conventional carrier sense and automatic gain control scheme.

When an STA is near an AP with a larger ACI, the RF part can be saturated, but the ADC is not saturated because the ACI is filtered out at the analog baseband lowpass filter stage. The conventional scheme, which does not have multi-channel multi-level carrier sensing and adaptive initial gain control functionality, shows performance degradation in this case. In contrast, the proposed IASO carrier sensor improves the dynamic range because it can avoid saturation of the RF/analog components and it reduces the false positive carrier sensing when the incoming signal includes a strong ACI. In the low data rate modes, the proposed IASO carrier sensor could not meet the system requirement for very large power incoming signals because the leakage power level from the interferer is larger than the high data rate modes because the ACI level is relatively higher. In this case, if the transmission has a $20 \mathrm{MHz}$ guard band (GB) between the ACI and desired signal, approximately $10 \mathrm{~dB}$ of additional gain can be achieved in a low data rate.

\subsection{Network emulation}

In the developed prototype, it is difficult to evaluate the system in dense network scenarios because it is necessary to have many hardware and software resources or expensive equipment. In order to overcome these problems, a software-based emulation environment can be used in order to reduce the evaluation cost and experimental setup time. Therefore, it is possible to emulate the hardware behavior and performance in the developed emulator. The hardware is manufactured using a hardware description language (HDL) in order to perform the synthesis, placement, and routing using various tools. Our FPGA prototype system was initially developed for verification using a hardwarelike $\mathrm{C}$ emulator. The emulator has been described with hardware architecture, and it has a cycle-true and bit-true description. That is, the emulator is programmed like a register transistor level (RTL) description model, and it has timing and bit width for all signals. This emulator was verified with a bit-matching process between the RTL and C model. The performance curves of the emulation are the same as the performance measurement results on the RTL targeted FPGA prototype system. In this way, we developed an emulation model to evaluate the proposed IASO scheme in dense networks.

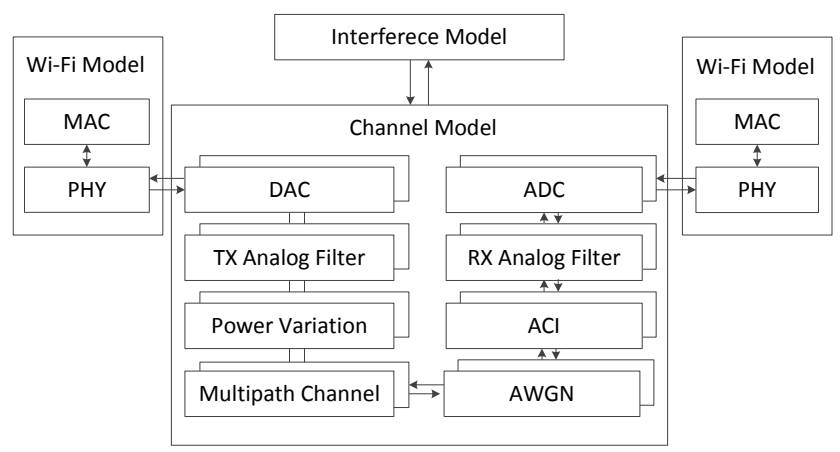

Figure 10. Emulation model.

As illustrated in Figure 10, there are three nodes in the emulation model: one AP, one station, and an interferer for the ACI. The impairments that are analyzed include the multipath channel, signal-to-noise ratio variation, and adjacent channel interference variation. The packet size is 1,000 bytes and the packet interval is $16 \mu \mathrm{s}$. The desired signal is $20 \mathrm{MHz}$ bandwidth mode at $5.3 \mathrm{GHz}$. The SNR and signal to ACI ratio vary randomly by additive white Gaussian noise (AWGN) and ACI models, respectively. The ACI is generated using a complex Gaussian process through a raised 
cosine filter that has a filter length of 24 taps and a rolloff factor of 0.2 . The ACI has $20 \mathrm{MHz}$ bandwidth at a $20 \mathrm{MHz}$ offset from the center frequency. In order to emulate the proposed scheme in a dynamically varying channel, a link adaptation scheme should be considered. The link adaptation scheme is essentially operated as an SNR-guided rate adaptation scheme in order to manage the high fluctuations of the SNR [23], and it adjusts the transmission rate adaptively to the varying channel conditions according to the adaptive auto rate fallback (ARF). This type of combined rate control is an effective method of improving the link quality under dynamically interfered varying channel conditions.

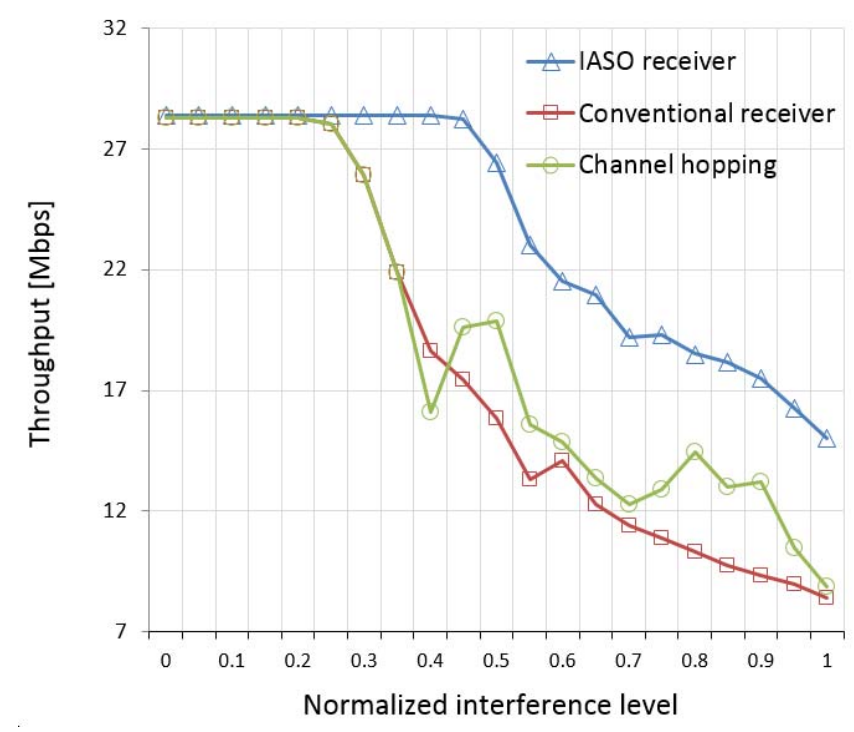

Figure 11. Throughput over the interference level.

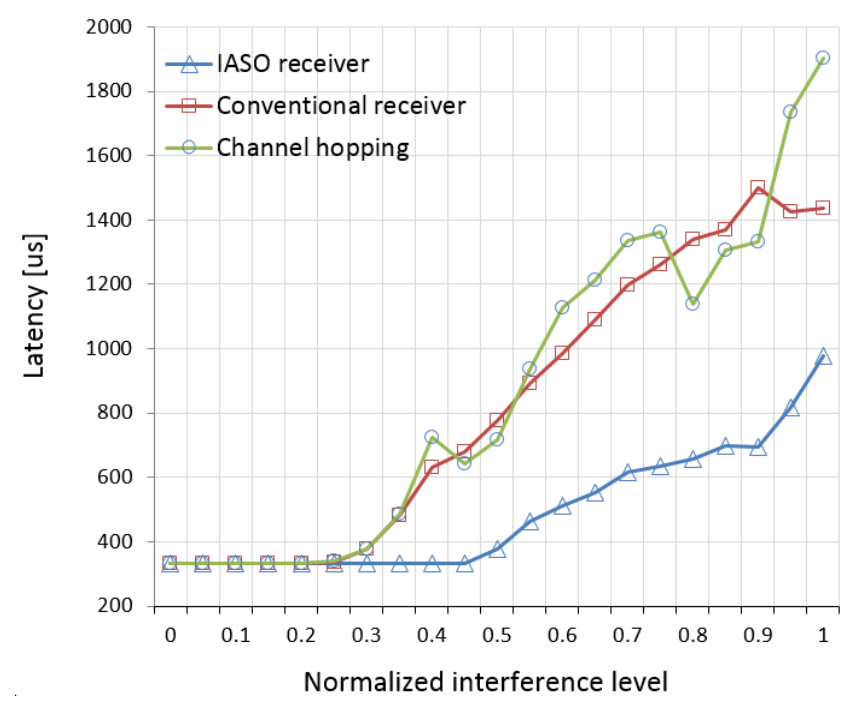

Figure 12. Latency over the interference level.

In Figures 11 and 12, the throughput and latency are plotted over randomly varying channels in order to compare the IASO scheme with the conventional scheme and channel hopping schemes. All cases are emulated in input power levels from $-50 \mathrm{dBm}$ to -90 $\mathrm{dBm}$ for the desired signal because the conventional scheme has a limited dynamic range in the receiver. As a result, they have the same throughput and latency performance when there is no interference in the channel. However, when the ACI level is larger than a specific level (i.e., 0.3), the proposed scheme exhibits improvement in terms of throughput and latency. For example, the IASO scheme shows throughput of approximately $10 \mathrm{Mbps}$ and latency improvement of $350 \mu \mathrm{s}$ at an interference level of 0.5 compared with the conventional scheme and channel hopping scheme, respectively. In this network emulation, the observed throughput was low because the input signal level was low and the channel conditions were significantly interfered with other nodes. The graphs demonstrate that the existing schemes decrease the throughput and increase the latency due to false carrier sensing or inappropriate gain control as the interference level increases beyond 0.3 .

In this network emulation, the channel hopping scheme was not effective because all channels randomly varied in terms of the interference and signal strength. When a channel was ideally static for a sufficiently long time period, the channel hopping scheme showed better performance at certain times than the IASO scheme. From the data presented in Table 2, the network emulation demonstrates that the IASO scheme improved the average throughput and latency by approximately $32 \%$ and $41 \%$, respectively, compared with the conventional schemes in dynamically varying interfered channel conditions. When compared with the channel hopping technique, the IASO scheme afforded $24 \%$ and $43 \%$ improvement in terms of average throughput and latency, respectively. The improvement results from the conventional carrier sense and gain control schemes not considering the ACI effect, and furthermore the channel hopping techniques require successive errors or throughput degradation for channel switching and cannot guarantee a good channel in the next hop. In addition, if the incoming signal power is larger than a certain level, the conventional schemes do not work because the dynamic range is limited due to the strong ACI. Therefore, the existing schemes are ineffective for dynamic interference; they consequently increase latency and decrease throughput.

Table 2. Evaluation summary

\begin{tabular}{|c|c|c|}
\hline \multirow{2}{*}{ Category } & \multicolumn{2}{|c|}{ Improvement over } \\
\cline { 2 - 3 } & Conventional receiver & Channel hopping \\
\hline Throughput & $32 \%$ & $24 \%$ \\
\hline Latency & $41 \%$ & $43 \%$ \\
\hline
\end{tabular}

\section{Conclusion}

In this paper, we analyzed the ACI effect and proposed IASO carrier sensing, a new ACI mitigation scheme for WLAN systems. In the existing literature, there has not yet been an investigation of adaptive initial gain control and multi-channel multi-level carrier sensing, or an implementation to mitigate the ACI effect in WLAN receivers. The proposed IASO scheme is designed to mitigate interference and provides substantial improvements in terms of the dynamic range of the receiver, throughput, and latency by enhancing the carrier sensor, and by utilizing the gain controller in networks with dense stations and BSSs that potentially interfere with each other. The FPGA prototype experiment and network emulation results confirm the 
superior performance of the IASO scheme in comparison with the existing schemes under dynamically varying interfered channel conditions. As future work, we plan to extend this work to combine the IASO scheme and dynamic sensitivity control for greater improvements under various channel conditions, and study an interference-aware power saving scheme to improve power efficiency of the receiver.

\section{ACKNOWLEDGMENTS}

Il-Gu Lee was supported in this work in part by the Korean government (MSIP and IITP) under grant 10044321. Myungchul Kim was supported by the Ministry of Science, ICT and Future Planning (MSIP), Korea, under the Human Resource Development Project for brain scouitng program (IITP-2015H7106-14-1011) supervised by the Institute for information \& communictions Technology Promotion (IITP).

\section{REFERENCES}

[1] G. Goth. 2012. Next-Generation Wi-Fi: As Fast as We'll Need? IEEE Internet Computing. 16, 6 (Nov. 2012), 7-9.

[2] Tae Hyun Kim, Jian Ni, R. Srikant, and N.H. Vaidya. 2013. Throughput-Optimal CSMA With Imperfect Carrier Sensing. IEEE/ACM Transaction on Networking. 21, 5 (Jan. 2013), 1636-1650.

[3] I. Harjula, J. Pinola, and J. Prokkola. 2011. Performance of IEEE 802.11 based WLAN devices under various jamming signals. In Proceedings of IEEE MILCOM, 2129-2135.

[4] W. Sun, O. Lee, Y. Shin, S. Kim, C. Yang, H. Kim, and S. Choi. 2014. Wi-Fi Could Be Much More. IEEE Communications Magazine. 52, 11 (Nov. 2014), 22-29.

[5] IEEE P802.11ac, Draft 7.0 (2013).

[6] Anatolij Zubow and Robert Sombrutzki. 2012. Adjacent Channel Interference in IEEE 802.11n. In Proceedings of IEEE WCNC, 1163-1168.

[7] M. Doering, L. Budzisz, D. Willkomm, and A. Wolisz. 2013. About the practicality of using partially overlapping channels in IEEE $802.11 \mathrm{~b} / \mathrm{g}$ networks. In Proceedings of IEEE ICC, 5110-5114.

[8] M.Sarper Gokturk, and Gulden Ferazoglu, 2014. Adjacent channel interference aware channel selection for wireless local area networks. In Proceedings of IEEE WCNC, 29222927.

[9] M.Y. Arslan, K. Pelechrinis, I. Broustis, S. Singh, S.V. Krishnamurthy, S. Addepalli, and K. Papagiannaki. 2013. ACORN: An Auto-Configuration Framework for 802.11n WLANs. IEEE/ACM Transaction on Networking. 21, 3 (Jun. 2013), 896-909.
[10] Shravan Rayanchu, Vivek Shrivastava, Suman Banerjee, and Ranveer Chandra. 2012. FLUID: Improving Throughputs in Enterprise Wireless LANs through Flexible Channelization. IEEE Transaction on Mobile Computing. 11, 9 (Sept. 2012), 1455-1469.

[11] Tianji Li, Mi Kyung Han, Apurv Bhartia, Lili Qiu, Eric Rozner, Yin Zhang, Brad Zarikoff. 2011. CRMA: collisionresistant multiple access. In Proceedings of ACM MobiCom, 61-72.

[12] Konstantinos Pelechrinis, Ioannis Broustis, Skikanth V. Krishnamurthy, and Christos Gkantsidis. 2011. A Measurement-Driven Anti-Jamming System for 802.11 Networks. IEEE/ACM Transaction on Networking. 19, 4 (Aug. 2011), 1208-1222.

[13] G. Smith. 2013. Dynamic Sensitivity Control. Standard document. IEEE 802.11-13/1012r4.

[14] Ramakrishna Gummadi, David Wetherall, Ben Greenstein, Srinivasan Seshan. 2007. Understanding and mitigating the impact of RF interference on 802.11 networks. In Proceedings of ACM SIGCOMM. 385-396.

[15] V.P.G. Jimenez, M.J. Fernandez-Getino Garcia, F.J.G. Serrano, and A.G. Armada. 2004. Design and Implementation of Synchronization and AGC for OFDMbased WLAN Receivers. IEEE Transaction on Consumer Electronics. 50, 4 (Nov. 2004), 1016-1025.

[16] IEEE Standard 802.11h (2003).

[17] Kunxiao Zhou, Xiaohua Jia, Liming Xie, Yanan Chang, and Xing Tang. 2012. Channel Assignment for WLAN by Considering Overlapping Channels in SINR Interference Model. In Proceedings of IEEE ICNC, 1005-1009.

[18] Bruno Kauffmann, Francois Baccelli, Augustin Chaintreau, Vivek Mhatre, Konstantina Papagiannaki, and Christophe Diot. 2007. Measurement-Based Self Organization of Interfering 802.11 Wireless Access Networks. In Proceedings of IEEE INFOCOM, 1451-1459.

[19] IEEE P802.11af, Draft 4.0 (2013).

[20] IEEE P802.11ah, Draft 2.0 (2014).

[21] Laurent Cariou, et al. 2013. High-efficiency WLAN. Standard document. IEEE 802.11-13/0331r5.

[22] Eldad Perahia, et al. 2014. High-efficiency WLAN overview. Standard document. IEEE 802.11-14/0214r2.

[23] Jiansong Zhang, Kun Tan, Jun Zhao, Haitao Wu, and Yongguang Zhang. 2008. A Practical SNR-Guided Rate Adaptation. In Proceedings of IEEE INFOCOM, 2083-2091. 